University of Nebraska - Lincoln

DigitalCommons@University of Nebraska - Lincoln

Papers in the Earth and Atmospheric Sciences Earth and Atmospheric Sciences, Department

7-1996

\title{
Boundary Conditions for Convergent Radial Tracer Tests and Effect of Well Bore Mixing Volume
}

Vitaly A. Zlotnik

University of Nebraska-Lincoln, vzlotnik1@unl.edu

John David Logan

University of Nebraska-Lincoln, jlogan1@unl.edu

Follow this and additional works at: https://digitalcommons.unl.edu/geosciencefacpub

Part of the Earth Sciences Commons

Zlotnik, Vitaly A. and Logan, John David, "Boundary Conditions for Convergent Radial Tracer Tests and Effect of Well Bore Mixing Volume" (1996). Papers in the Earth and Atmospheric Sciences. 159.

https://digitalcommons.unl.edu/geosciencefacpub/159

This Article is brought to you for free and open access by the Earth and Atmospheric Sciences, Department of at DigitalCommons@University of Nebraska - Lincoln. It has been accepted for inclusion in Papers in the Earth and Atmospheric Sciences by an authorized administrator of DigitalCommons@University of Nebraska - Lincoln. 


\title{
Boundary conditions for convergent radial tracer tests and effect of well bore mixing volume
}

\author{
Vitaly A. Zlotnik \\ Department of Geology, University of Nebraska at Lincoln \\ J. David Logan \\ Department of Mathematics, University of Nebraska at Lincoln
}

\begin{abstract}
Convergent radial flow tracer tests have a complex spatial nonaxial transport structure caused by the flow in the vicinity of the injection well and its finite mixing volume. The formulation of the boundary value problem, and especially the treatment of the boundary conditions at the injection well, is nontrivial. Hodgkinson and Lever [1983], Moench [1989, 1991], and Welty and Gelhar [1994] have developed different models and methods for the analysis of breakthrough curves in the extraction well. To extend interpretation techniques to breakthrough curves in the zone between injection and extraction wells, an analysis of conventional transport models is given, and improved boundary conditions are formulated for a convergent radial tracer test problem. The formulation of the boundary conditions is based upon a more detailed analysis of the kinematic flow structure and tracer mass balance in the neighborhood of the injection well. Two practical applications of revised boundary conditions for field data analysis are given. First, the note explains anomalous high well bore mixing volumes of injection wells found by Cady et al. [1993] and allows one to establish the role of mixing versus other processes (retardation, matrix diffusion, etc.). Second, it is shown that the improper use of Moench's [1989] model can produce bias in the characteristics of breakthrough curves in the extraction well under conditions that involve a significant mixing factor in the injection well. A numerical example indicates an error in peak concentrations on a breakthrough curve by as much as $70 \%$ and in peak arrival time by $10 \%$ for Peclet numbers $P e=10^{2}$. The effect becomes slightly less significant for $P e=1$.
\end{abstract}

\section{Introduction}

The advantages and disadvantages of convergent radial flow tracer tests have been documented in different studies and different countries, especially with regard to high-level nuclear waste disposal [Gelhar et al., 1992, Luckner and Shestakov, 1991] or organic pollutants [Mackay et al., 1994]. Usually, the studies and experiments are limited to the analysis of breakthrough curves in pumping wells, although new sampling technologies (multilevel samplers) are available for concentration measurements at arbitrary points in the field [Mackay et al., 1994]. Because of the complexity of the model, the analysis is often reduced to adjusted one-dimensional solutions [Thorbjarnson and Mackay, 1994] instead of a more accurate twodimensional transport model. Unlike diverging flow tracer tests, the converging flow structure does not have axial symmetry, especially in the vicinity of the injection well, and therefore formulation of the boundary conditions is a difficult problem.

Hodgkinson and Lever [1983], Moench [1989, 1991], and Welty and Gelhar [1994] have developed different models for the analysis of convergent flow tracer tests and outlined the differences from divergent flow tracer tests. Hodgkinson and Lever [1983] considered nonaxisymmetric transport between injection and extraction wells in fractured media. Their goal

Copyright 1996 by the American Geophysical Union.

Paper number 96WR01103.

0043-1397/96/96WR-01103\$09.00 was the analysis of breakthrough curves in an extraction well, which was found after a series of simplifications from the solution of the axisymmetric problem. Hydrodynamic dispersion was approximated by quadratic dependence of the dispersion coefficient on the seepage velocity in a fracture. Finally, a numerical solution of the problem was obtained by application of the modified Talbot [1979] algorithm for Laplace transform inversion. This approach is not directly applicable to unconsolidated uniform materials, although it properly accounts for differences in geometry between convergent and divergent flow tracer experiments.

Moench [1989, 1991] developed a method for interpreting breakthrough curves in homogeneous media using the solution of the axisymmetric problem again obtained after a series of simplifications. Longitudinal hydrodynamic dispersion was approximated by a linear function of seepage velocity. Experiments with numerical Laplace transform inversions by Talbot [1979] and de Hoog et al. [1982] indicated high efficiency of the algorithm for a wide range of Peclet numbers. Again, the operational approach was reduced to analysis of breakthrough curves in an extraction well. Moench's method is supported by a variety of software.

Recently, Welty and Gelhar [1989, 1994] published another method for interpreting breakthrough curves based on a perturbation method by Gelhar and Collins [1971]. They also reduced the problem to an axisymmetric one. The approach can possibly be extended to nonaxisymmetric problems for finding tracer concentrations in the aquifer, but use of perturbation 


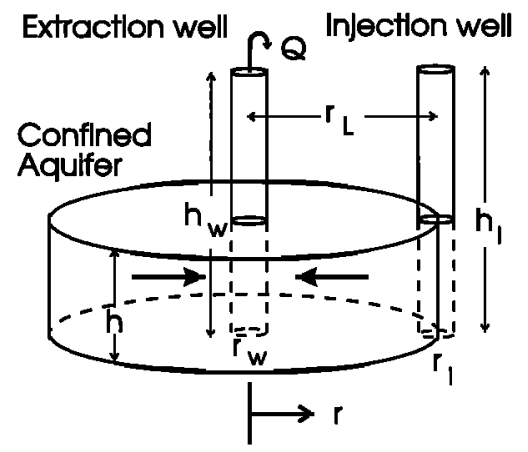

Figure 1. Schematic diagram of convergent flow tracer test.

methods for boundary values problem with two variables may involve complexities beyond the straightforward methods of integral transform inversion.

The approach by Hodgkinson and Lever [1983] and Moench [1989] appears to be a more direct method for treating convergent flow tracer experiments because their analyses do not significantly alter the transport equation. The method can be extended to solving the nonaxisymmetric problem, which is needed for a thorough understanding of the concentration field in the aquifer. However, a careful analysis shows that these two models treat boundary conditions differently for similar geometries.

In this note these differences will be reconciled, and recommendations for an improved analysis will be given.

\section{Boundary Condition at the Injection Well}

A difficulty with the boundary condition at the injection well is evident in existing studies. To approximate effects of mixing in the injection well, Moench [1989, p. 441] used the hypothesis that the average value of the tracer concentration over a large cylindrical surface centered at the extraction well that passes through the injection well equals the tracer concentration in the injection well. Specifically, if the injection well is at a distance $r_{L}$ from the center of the extraction well, then it is presumed that $C\left(r_{L}, t\right)=c_{I}(t)$, where $C\left(r_{L}, t\right)$ is the average concentration over the cylindrical surface of radius $r_{L}$ and height $h$ (the aquifer saturated thickness) and $c_{I}(t)$ is the concentration of the tracer in the perfectly stirred mixture in the injection well (Figure 1). This condition is obvious for divergent flow tracer tests.

However, a careful analysis of the flow field in the vicinity of the well shows that this condition can lead to errors in some cases of convergent flow tracer tests. We shall comment on these cases in section 5 . We proceed now with the formulation of the boundary condition, retaining Moench's [1989] notation where possible.

\subsection{Concentration-Based Boundary Condition}

The origin of polar coordinates $(r, \theta)$ is located at the center of the extraction well of radius $r_{W}$, which has tracer mixing length $h_{W}$. The concentration distribution $c(r, \theta, t)$ is not an axisymmetric function owing to the presence of the injection well of radius $r_{I}$ and mixing length $h_{I}$ with center located at the point $\left(r_{L}, 0\right)$. The injection and extraction wells have screens of length $h$ located at the same depths. Similar to Sauty [1980], we introduce an azimuthally averaged concentration defined by

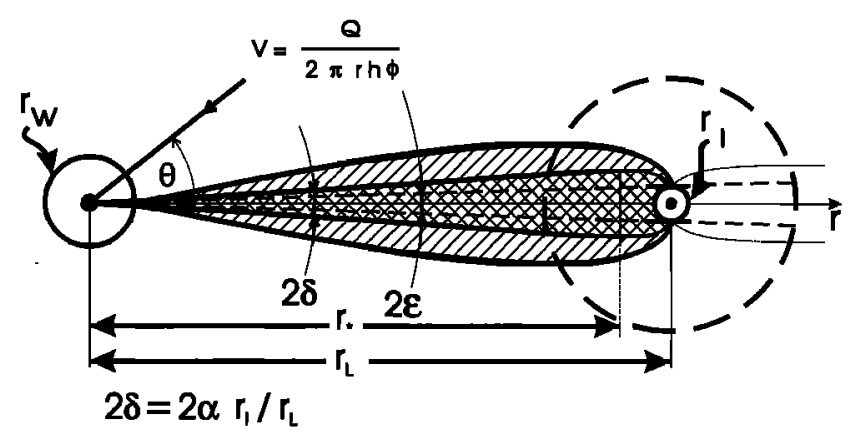

QI7/ advectlon and dispersion

\section{advection dominated zone}

Figure 2. Plane view of convergent flow tracer test.

$$
C(r, t)=\frac{1}{2 \pi} \int_{-\pi}^{+\pi} c(r, \theta, t) d \theta
$$

Let us consider the aperture angle $2 \varepsilon=2 r_{I} / r_{L}$ between two rays originating in the center of extraction well and tangent to the circumference of injection well. A plane view is shown in Figure 2. Since $2 r_{l} \ll r_{L}$, the aperture is small. We now observe that streamlines in the vicinity of the injection well at distances larger than a few injection well diameters are the same as in the case of uniform, horizontal ambient flow (Figure 3 ). The seepage velocity $V$ of the ambient radial flow near the injection well caused by extraction is computed from the formula

$$
V(r)=-Q /(2 \pi h r \phi), r \approx r_{L}
$$

where $\phi$ is effective porosity (Figures 2 and 3). Because the injection well circumference is considered as a boundary of constant head, this velocity field (2) will be distorted by the mere presence of the injection well, even with zero flow rate of injection. Only case of an infinitesimal flow rate of water injection is considered herein; for finite flow rates a different approach is needed [Guvanasen and Guvanasen, 1987].

The case of a well without pumping in the ambient horizon-

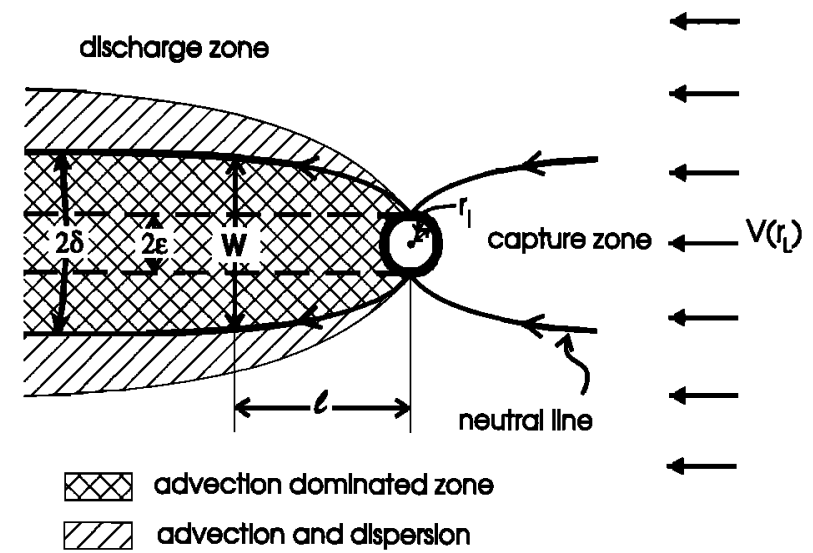

Figure 3. Local disturbance of velocity field in the vicinity of the injection well with zero flow rate. Ambient flow has velocity $V\left(r_{L}\right) \approx-Q /\left(2 \pi r_{L} h \phi\right)$. 


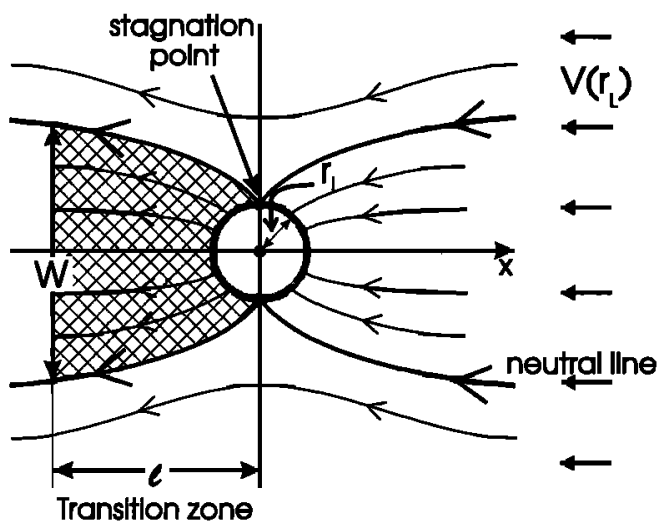

Figure 4. Schematic view of capture and discharge zones in the vicinity of the injection well.

tal uniform flow was studied by Ogilvy [1958], Drost et al. [1968], Luckner and Shestakov [1991], and Bidaux and Tsang [1991]. The maximal width of the capture zone, or the discharge zone, near the injection well is $w=2 \alpha r_{I}$ (Figure 4), where $\alpha$ is a factor that defines the distortion of distance between the two most separated streamlines entering (or leaving) the injection well (this parameter can also depend on skin effect for an injection well). For uniform isotropic aquifer with a well without skin, $\alpha=2$. For skins with higher conductivity, $2 \leq \alpha \leq 4$, and for skins with lower conductivity $0<\alpha \leq 2$ [Drost et al., 1968]. The width of the capture zone or the discharge zone varies from $w=2 r_{I}$ at the injection well to $95 \%$ of the width $w=2 \alpha r_{I}$ within distance of less than $5 r_{I}$ upstream and downstream from the injection well, respectively; i.e., the length of the transitional zone is $l \approx 5 r_{l}$. The aperture angle of this narrow zone at the distance $r_{*} \approx r_{L}-$ $l$ from the center of extraction well is

$$
2 \delta=2 \alpha r_{I} / r_{L}
$$

The simplification of replacing slightly convergent radial flow toward an extraction well with a uniform, horizontal, flow in the vicinity of injection is a geometrical simplification (Figures 2 and 3). The estimate of $\alpha$ can be improved by considering a nonzero aperture angle $2 \varepsilon$ of ambient radial flow. However, the correction value is of the order of an aperture angle and therefore can be safely neglected.

To derive the boundary condition at the injection well, we consider flow and transport in a ring-shaped domain centered at the extraction well and bounded by circles of radii $r=r_{W}$ and $r_{*}=r_{L}-l\left(l \ll r_{L}\right)$. The physical assumption is that at a small distance $l$ downstream in the discharge zone of the injection well, advective transport is dominant over dispersive transport. (This is true for injection with a zero flow rate that does not disturb flow in the immediate vicinity of the borehole.)

Therefore the concentration distribution $c(r, \theta, t)$ at a distance $r=r_{*}$ from the extraction well is

$$
c(r, \theta, t)=\left\{\begin{array}{cl}
c_{I} & 0 \leq|\theta|<\delta \\
0 & \delta<\theta<\pi
\end{array}\right.
$$

where $c_{I}$ is the concentration generated in the injection well and transported downstream through the narrow (width $w$ ) and short (a few well diameters) discharge zone by advection. This small zone with advection dominated flow has aperture angle $2 \delta$ (Figures 2 and 3). Using definition (1), the boundary condition for the azimuthally averaged concentration is therefore given by

$$
C\left(r_{*}, t\right)=\frac{\delta}{\pi} c_{I}
$$

This boundary condition differs from the one given by Moench [1989].

It remains to determine the effluent concentration from the injection well in an ambient horizontal flow field with velocity $V\left(r_{L}\right)$. This problem has been studied with regard to the borehole dilution method [Ogilvy, 1958; Drost et al., 1968; Freeze and Cherry, 1979; Luckner and Shestakov, 1991]. Effluent concentration from the well with initial dissolved tracer mass $M_{0}$ and with constant tracer mass injection $\dot{M}$ to the well (per unit time) satisfies a mass balance equation for the tracer in borehole, namely,

$$
-2 \alpha r_{I} \phi h\left|V\left(r_{L}\right)\right| c_{I}=\pi r_{I}^{2} h_{I} \frac{d c_{I}}{d t}-\dot{M} \quad c_{I}(0)=\frac{M_{0}}{\pi r_{I}^{2} h_{I}}
$$

Integration of this linear differential equation is straightforward in the case of the injection of a tracer slug $\left(M_{0}>0, \dot{M}\right.$ $=0)$ or in the case of continuous injection $\left(M_{0}=0, \dot{M}>0\right)$. After integration, the known effluent concentration $c_{I}(t)$ can be substituted into boundary conditions (4) and (5).

\subsection{Mass-Flux-Based Boundary Condition}

The boundary condition for mass flux at the boundary $r=$ $r_{*}$ can also be derived from mass conservation [Novakowski, 1992]. It is assumed that the advective-dispersive mass flux to the transport domain $r_{W}<r<r_{*}$ is equal to the advective mass flux from injection well, that is,

$$
-D_{r} \frac{\partial c}{\partial r}+V c=\left\{\begin{array}{ll}
V c_{I} & 0 \leq|\theta|<\delta \\
0 & \delta<|\theta|<\pi
\end{array} \quad r=r_{*} \approx r_{L}\right.
$$

where $D_{r}$ is the coefficient of longitudinal (radial) dispersion, which is determined by the longitudinal dispersivity $\alpha_{L}$ and is given by $D_{r}=\alpha_{L}|V|$. Using (1), (4), and (7), one immediately obtains the boundary condition for $C(r, t)$

$$
-D_{r} \frac{\partial C}{\partial r}+V C=\frac{\delta}{\pi} V c_{l} \quad r=r_{*} \approx r_{L}
$$

which relates $C\left(r_{*}, t\right)$ at the boundary with the concentration of effluent from the injection well.

Boundary conditions (7) and (8) have obvious physical consequences. Condition (7) implies a nonuniform distribution of specific mass flux over a circle of radius $r=r_{*}$. Condition (8) indicates that the total mass entering the circle of radius $r_{L}$ is exactly equal to the amount of mass discharged by the injection well.

The effluent concentration $c_{I}$ needed in boundary condition (7) or (8) is available from the solution of equation (6). Thus boundary conditions (7) or (8) (after solution of equation (6) for the effluent concentration) constitute an alternate set of boundary conditions, in lieu of (4) or (5).

\subsection{Concentration-Mass Flux Condition for Azimuthally Averaged Concentration}

As will be shown below, only the azimuthally averaged concentration is required when the analysis of breakthrough curves is used for interpreting tracer tests. This was shown 
accurately by Hodgkinson and Lever [1983] and used implicitly by Moench [1989]. In addition, Moench [1989] restricted the transport domain to a finite radius $r_{L}$ instead of the infinite domain considered by Hodgkinson and Lever [1983].

In our study we proceed in two steps. First, we substitute the effluent concentration $c_{I}$ obtained from condition (6) into (8) to obtain

$$
2 \pi r_{L} \phi h\left[-D_{r} \frac{\partial C}{\partial r}+V C\right]_{r=r_{.}}=\pi r_{I}^{2} h_{l} \frac{\partial c_{I}}{\partial t}-\dot{M}
$$

Second, the boundary condition (5) can be incorporated into this equation to get

$2 \pi r_{L} \phi h\left[-D, \frac{\partial C}{\partial r}+V C\right]_{r=r_{*}}=\left.\pi r_{I}^{2} h_{I} \frac{\pi}{\delta} \frac{\partial C}{\partial t}\right|_{r=r_{*}}-\dot{M}$

We observe that this boundary condition does not include the effluent concentration for the injection well. Moreover, it differs from Moench's [1989] boundary condition by a large factor $\pi / \delta \gg 1$ in front of the time derivative.

\section{Boundary Condition at the Extraction Well}

The boundary condition at the extraction well screen for perfect mixing is

$$
c\left(r_{W}, \theta, t\right)=c_{W}(t) \quad 0 \leq \theta<2 \pi
$$

where $c_{W}(t)$ is the concentration of the tracer in the borehole at the well screen circumference. An additional equation for determining the concentration $c_{W}(t)$ can be obtained from tracer mass balance in the well [Novakowski, 1992], namely,

$$
\pi r_{W}^{2} h_{W} \frac{d c_{W}(t)}{d t}=\phi h \int_{0}^{2 \pi}\left[D, \frac{\partial c}{\partial r}-V c\right]_{r=r W} r_{w} d \theta-Q c_{w}(t)
$$

These two equations are sufficient for well-posedness of the boundary value problem. To our knowledge, these boundary conditions have not been noted previously.

A single boundary condition for $C$ can now be obtained from these equations. From (1) it follows that

$$
C\left(r_{W}, t\right)=c_{W}(t)
$$

Thus the concentration in the extraction well is equal to the average concentration in the aquifer around the well. Next, using (1) and (2), equations (11) and (12) can be reduced to a single boundary condition

$$
\pi r_{W}^{2} h_{W} \frac{\partial C}{\partial t}=2 \pi r_{w} \phi h D_{r} \frac{\partial C}{\partial r} \quad r=r_{W}
$$

In different forms, this or a similar condition has been suggested and used by different authors [Hodgkinson and Lever, 1983; Moench, 1989; Luckner and Shestakov, 1991].

\section{Transport Equation}

For completeness we write down the equation for mass transport between the injection and extraction wells. The flow is two dimensional under the condition that both wells have screens at the same elevation and of the same length. In this case the transport equation in cylindrical coordinates without $z$ derivatives [Bear, 1979 , p. 246 , equation $7-58]$ is
Table 1. Dimensionless Parameters

\begin{tabular}{ll}
\hline \multicolumn{1}{c}{ Dimensionless Quantity } & \multicolumn{1}{c}{ Expression } \\
\hline Time & $t_{D}=\frac{Q t}{\pi h \phi\left(r_{L}^{2}-r_{W}^{2}\right)}$ \\
Distance & $r_{D}=\frac{r}{r_{L}}$ \\
Extraction well radius & $r_{W D}=\frac{r_{W}}{r_{L}}$ \\
Peclet number & $P e=\frac{r_{L}}{\alpha_{L}}$ \\
Concentration (slug input) & $C_{D}=\frac{C}{M_{0} /\left[\pi h \phi\left(r_{L}^{2}-r_{W}^{2}\right)\right]}$ \\
Concentration (continuous input) & $C_{D}=\frac{C}{\dot{M} / Q}$ \\
Extraction well mixing factor & $\mu_{W}=\frac{r_{W}^{2} h_{W}}{\phi h\left(r_{L}^{2}-r_{W}^{2}\right)}$ \\
Injection well mixing factor (revised) & $\mu_{J}=\frac{\pi r_{I} r_{L} h_{I}}{\alpha \phi h\left(r_{L}^{2}-r_{W}^{2}\right)}$ \\
\hline
\end{tabular}

$$
R \frac{\partial c}{\partial t}=\frac{1}{r} \frac{\partial}{\partial r}\left(r D_{r} \frac{\partial c}{\partial r}\right)+\frac{1}{r^{2}} \frac{\partial}{\partial \theta}\left(D_{\theta} \frac{\partial c}{\partial \theta}\right)-V \frac{\partial c}{\partial r}
$$

where $R$ is retardation factor and $D_{\theta}$ is coefficient of transverse dispersion. It can be expressed by transverse dispersivity $\alpha_{T}$ as follows: $D_{\theta}=\alpha_{T}|V|$. After azimuthal averaging, with consideration of the $2 \pi$ periodicity of $c$ and $\partial c / \partial \theta$, the simple axisymmetric equation for concentration $C$ becomes

$$
R \frac{\partial C}{\partial t}=\frac{1}{r} \frac{\partial}{\partial r}\left(r D, \frac{\partial C}{\partial r}\right)-V \frac{\partial C}{\partial r}
$$

\section{Applications of the Derived Boundary Condition}

5.1. Solution With Concentration-Mass Flux Boundary Condition

According to (5), the azimuthally averaged concentration at the radial distance $r_{L}$ is not equal to concentration of effluent from the injection well. After comparison of boundary condition (10) with Moench's [1989] equation (6), two features are apparent: (1) the coefficient in front of time derivative $\partial C / \partial t$ differs by a factor $\pi / \delta$, and (2) the condition (10) is universal for instantaneous or continuous tracer injection with a zero flow rate. In a typical example, $r_{I}=0.05 \mathrm{~m}, r_{L}=10 \mathrm{~m}$, and $\alpha \approx 2$, which means that $\pi / \delta \approx 300$. Therefore a revision to the prior solution of the problem for the breakthrough concentration in the extraction well is needed to account properly for finite injection well mixing volume.

The axisymmetric boundary value problem (16), (14), and (10), with initial averaged concentration $C(r, 0)=0, r_{W}<r$ $<r_{L}$, allows one to determine the breakthrough concentration without resorting to the solution of the boundary value problem for the concentration $c(r, \theta, t)$. This approach was used by Hodgkinson and Lever [1983] and Moench [1989], who simplified the problem and derived breakthrough curve concentrations using semianalytical methods involving numerical Laplace transforms.

Following Moench [1989], we first nondimensionalize the problem using dimensional parameters shown in Table 1. After dimensionless variables are introduced, our boundary value problem (equations (16), (14), and (10), with initial averaged 
concentration $\left.C(r, 0)=0, r_{W}<r<r_{L}\right)$ becomes completely equivalent to that of Moench [1989, equations (9)(13)]. The only revision involves the injection well mixing factor $\mu_{I}$. Revision is achieved by multiplying Moench's [1989, Table 1] injection well mixing factor by a multiplier $\pi / \delta$. The unrevised parameter $\mu_{I}$ underestimates the mixing volume of the injection well by a factor $\pi / \delta$, which is of a few orders of magnitude. In the example given above, the parameter $\mu_{I}$ differs by a factor of 300 .

Therefore the numerical solution of Moench [1991] can still be used. The method was intensively tested versus finitedifference and approximate analytical methods, and it proved to be highly accurate and stable for a wide range of Peclet numbers.

\subsection{Practical Significance of Corrections}

We now address the natural question regarding the practical significance of the correction presented above.

Theoretically, Moench's [1989, 1991] solution in dimensional variables was verified mainly for zero mixing volume of injection and extraction wells. For nonzero mixing well volumes, the solution in dimensionless variables was tested by comparing it with finite-difference solutions, and discrepancies could not be detected. Other tests compared the dimensionless solution with the one by Gelhar and Collins [1971] for zero mixing volume.

This method has been routinely applied to various field studies. Recently, Cady et al. [1993] used this method for processing convergent radial flow tracer tests, and they attempted to fit breakthrough curves to Moench's solution. Unexpectedly, they found "... high mixing factors required to fit the breakthrough curves" and concluded that using "... the formula provided by Moench [1989], the mixing factor for the tracer injection well should be 2 to 3 orders of magnitude smaller than reported here" [Cady et al., 1993, p. 2981]. Two possible explanations were offered: (1) the method of estimating of well mixing factor should be revised, (2) diffusion into the matrix should be considered.

Our results explain their field data. In the experiment the injection well radius was $r_{I}=0.08 \mathrm{~m}$, and $r_{L}$ was $19.8 \mathrm{~m}$ and $22.3 \mathrm{~m}$ in two different experiments (S. Silliman, personal communication, 1995). Using the shape factor $\alpha=2$, we obtain a correction factor $\pi / \delta \approx 400$ needed for their test geometry. This value supports experimental findings by Cady et al. [1993] on the role of well bore mixing and indicates that the hypothesis of diffusion into the matrix is not needed.

Another illustration of the significance of the proposed correction can be shown using numerical examples. Our examples are based on the dimensionless results published by Moench [1989, Figure 5b]. This figure compares breakthrough curves in the extraction well $\left(\mu_{W}=0.05\right)$ produced by two different injection wells $\left(\mu_{I}=0.01\right.$ and $\mu_{I}=0.25$ ) for different Peclet numbers $P e=\left|V\left(r_{L}\right)\right| r_{L} / D_{r}=r_{L} / \alpha_{L}$. A 25-fold decrease of $\mu_{I}$ from $\mu_{I}=0.25$ to $\mu_{I}=0.01$ produced increase of peak concentration on a breakthrough curve by approximately $70 \%$ and accelerated the peak arrival time by at least $10 \%$ for $P e=$ 100. The effect becomes less significant for $P e=1$ under the same 25-fold increase in the injection well mixing factor.

Assuming that $\mu_{I}=0.01$ was obtained by using the unrevised formula and $\mu_{T}=0.25$ was obtained using the revised formula in Table 1, one arrives at the conclusion that the correction suggested by our analysis of the boundary condition cannot be neglected.
These two values of mixing factors for the injection well can be representative of small scale tracer tests where $r_{I}=0.05 \mathrm{~m}$, $r_{W}=0.0625 \mathrm{~m}, r_{L}=1.25 \mathrm{~m}, \phi=0.16$, and $\alpha=3.14$. The longitudinal dispersivity value $\alpha_{L}=0.0125 \mathrm{~m}$ is common for this test scale [Gelhar et al., 1992] which gives $P e=100$. Lower values of the Peclet number $(P e=1)$ require a much higher degree of aquifer heterogeneity $\left(\alpha_{L}=1.25 \mathrm{~m}\right)$ than is observed in natural or laboratory conditions.

Consequently, use of the unrevised formula for $\mu_{I}$ for parameter identification in Table 1 can produce a bias for injection wells with significant volume. The magnitude of the correction depends on the value of $r_{I} / r_{L}$, and in field scale experiments one may expect even larger differences. Using the uncorrected mixing factor for the injection well is equivalent to underestimating the mixing volume of the injection well. This conclusion may have serious implications in interpreting field tracer tests. In analyzing field experiments with the unrevised $\mu_{l}$, one finds that the injection well actually releases the tracer mass slower than is predicted by modeling. Then one can mistakenly attribute the apparent tracer retardation observed in field experiments to nonexisting processes involved in transport (reactions on surfaces, diffusion to matrix, etc.). In reality, it is an artifact of underestimating the injection well mixing factor.

Additional analysis of role of well bore mixing has been given by Welty and Gelhar [1994].

\section{Conclusions}

An analysis of conventional transport models for a convergent radial tracer test is given, and general boundary conditions are formulated. The formulation is based upon analysis of the kinematic flow structure and tracer mass balance in the neighborhood of the injection well.

An improved boundary condition for an azimuthally averaged concentration was derived based on a detailed analysis of flow and advective transport in the vicinity of injection well. The condition is important for the valid interpretation of breakthrough curves in the extraction well involving mixing in the injection well.

It is shown that boundary conditions at the injection well that underestimate the well mixing volume can significantly alter breakthrough curves in the extraction well (peak values and peak arrival time). A numerical example indicates an error in peak concentrations on a breakthrough curve by as much as $70 \%$ and in peak arrival time by $10 \%$ for Peclet numbers $P e=$ $10^{2}$. The effect becomes slightly less significant for $P e=1$. Therefore the term involving well bore mixing in Moench's [1989] boundary condition is revised using mass balance between the injection well and the aquifer. The unrevised injection well mixing factor may introduce such artifacts as nonexistent physical and chemical processes in order to achieve a fit between the model and field data.

These results explain the anomalous high well bore mixing volumes of injection wells found experimentally in various field studies [e.g., Cady et al., 1993].

After recalculation of the dimensionless injection well mixing factor $\mu_{I}$ according to the recommendations above, the Moench [1989, 1991] method with the Laplace inversion algorithm can be applied as before using existing software.

Acknowledgments. This work was partially supported by the Nebraska Central Platte NRD, Nebraska Research Initiative, and UNL 
Research Council. The authors are grateful to Allen F. Moench (U.S. Geological Survey), whose meticulous review of the early version of the manuscript and suggestions significantly improved this note. The authors acknowledge comments and data from J. J. Butler Jr., D. P. Hodgkinson, K. S. Novakowski, S. E. Silliman, and C. Welty on interpretation of convergent tracer tests.

\section{References}

Bear, J., Hydraulics of Groundwater, McGraw-Hill, New York, 1979. Bidaux, P., and C.-F. Tsang, Fluid flow around a well bore or an underground drift with a complex skin effects, Water Resour. Res., 27(11), 2993-3008, 1991.

Cady, C. C., S. E. Silliman, and E. Shaffern, Variation in aperture estimate ratios from hydraulic and tracer tests in a single fracture, Water Resour. Res., 29(9), 2975-2982, 1993.

de Hoog, F. R., J. H. Knight, and A. N. Stokes, An improved method of Laplace transforms using a Fourier series approximation, SLAM J. Sci. Stat. Comput., 3(3), 357-366, 1982.

Drost, W., D. Klotz, A. Koch, H. Moser, F. Neumaier, and W. Rauert, Point dilution methods of investigating ground water flow by means of radioisotopes, Water Resour. Res., 4(1), 125-146, 1968.

Freeze, R. A., and J. A. Cherry, Groundwater, Prentice-Hall, Englewood Cliffs, N. J., 1979.

Gelhar, L. W., and M. A. Collins, General analysis of longitudinal dispersion in nonuniform flow, Water Resour. Res., 7(6), 1511-1521, 1971.

Gelhar, L. W., C. Welty, and K. R. Rehfeldt, A critical review of data on field-scale dispersion in aquifers, Water Resour. Res., 28(7), 1955$1974,1992$.

Guvanasen, V., and V. Guvanasen, An approximate solution for tracer injection tests in a confined aquifer with a radially converging flow and finite volume of tracer and chase fluid, Water Resour. Res., 23(8), $1607-1619,1987$.

Hodgkinson, D. P., and D. A. Lever, Interpretation of a field experiment on transport of sorbed and non-sorbed tracers through a fracture in crystalline rock, Radioact. Waste. Manage. Nucl. Fuel Cycle, 4(2), 129-158, 1983 .

Luckner, L., and V. Shestakov, Migration Processes in the Soil and Groundwater Zone, Lewis, Boca Raton, Fla., 1991.
Mackay, D. M., G. Bianchi-Mosquera, A. W. Kopania, and H. Kianjah, A forced-gradient experiment on solute transport in the Borden aquifer, 1, Experimental methods and moment analysis of results, Water Resour. Res., 30(2), 369-383, 1994.

Moench, A., Convergent radial dispersion: A Laplace transform solution for aquifer testing, Water Resour. Res., 25(3), 439-447, 1989.

Moench, A., Convergent radial dispersion: A note on evaluation of the Laplace transform solution, Water Resour. Res., 27(12), 3261-3264, 1991.

Novakowski, K. S., An evaluation of boundary conditions for onedimensional solute transport, 1, Mathematical development, Water Resour. Res., 28(9), 2399-2410, 1992.

Ogilvy, N. A., An electrolyte method of determining the seepage velocity of ground water (in Russian), Byull. Nauchno Tekh. Inf., 4(16), 25-40, 1958.

Sauty, J.-P., An analysis of hydrodispersive transfer in aquifers, Water Resour. Res., 16(1), 145-158, 1980.

Talbot, A., The accurate numerical inversion of Laplace transforms, $J$. Inst. Math. Its Appl., 23, 97-120, 1979.

Thorbjarnson, K., and D. M. Mackay, A forced gradient experiment on solute transport in the Borden aquifer, 2, Transport and dispersion of the conservative tracer, Water Resour. Res., 30(2), 385-399, 1994.

Welty, C., and L. W. Gelhar, Evaluation of longitudinal dispersion from tracer test data, Rep. 320, Ralph M. Parsons Lab. for Water Res. and Hydrodyn., Dep. of Civ. Eng., Mass. Inst. of Technol., Cambridge, 1989.

Welty, C., and L. W. Gelhar, Evaluation of longitudinal dispersivity from non-uniform flow tracer tests, J. Hydrol., 153, 71-102, 1994.

J. D. Logan, Department of Mathematics, University of Nebraska at Lincoln, Lincoln, NE 68588-0323.

V. A. Zlotnik, Department of Geology, 214 Bessey Hall, P.O. Box 880340, University of Nebraska at Lincoln, Lincoln, NE 68588-0340. (e-mail: phrdvz@engvms.unl.edu)

(Received November 27, 1995; revised March 25, 1996; accepted April 8, 1996.) 